\title{
Development of antioxidant food packaging materials with controlled release properties
}

\author{
Seyhun Gemili ${ }^{a}$, Ahmet Yemenicioğlu ${ }^{b}$, Sacide Alsoy Altınkaya ${ }^{a, *}$ \\ a Department of Chemical Engineering, Faculty of Engineering, Izmir Institute of Technology, Gülbahçe Köyü, 35430 Urla, Izmir, Turkey \\ ${ }^{\mathrm{b}}$ Department of Food Engineering, Faculty of Engineering, Izmir Institute of Technology, Gülbahçe Köyü, 35430 Urla, Izmir, Turkey
}

\section{A R T I C L E I N F O}

\section{Article history:}

Received 20 March 2009

Received in revised form 6 August 2009

Accepted 8 August 2009

Available online 23 August 2009

\section{Keywords:}

Antioxidant packaging

L-tyrosine

L-ascorbic acid

Controlled release

Cellulose acetate

\begin{abstract}
A B S T R A C T
In this study, cellulose acetate (CA) films with different morphological features were prepared in order to control the release rates of low molecular weight natural antioxidants, L-ascorbic acid and L-tyrosine. Increasing CA content in the casting solution decreased the average pore size and porosity of the films, thus, reduced the diffusion rates of both antioxidants through the films. Although both antioxidants have similar molecular weights, L-tyrosine released into water much more slowly than L-ascorbic acid. The highest antioxidant activity in release test solutions was observed with highly porous L-tyrosine containing films. However, when the porosity of the films reduced, the antioxidant activity of L-ascorbic acid released into solution was found to be higher due to trapping of significant amount of L-tyrosine in dense films. The use of different antioxidants caused different changes in morphological and mechanical properties of the CA films. Varying the structural features of the films with the preparation conditions or using different surfaces of the films allowed the controlled release of each antioxidant.
\end{abstract}

(c) 2009 Elsevier Ltd. All rights reserved.

\section{Introduction}

Traditionally, antimicrobials or antioxidants are mixed into initial food formulations, or alternatively foods are dipped into solutions containing these additives. Limitations of these approaches are: the cease of protection effect once the active compounds are consumed in complex reactions of food system and lack of selectivity to target the food surface where most microbial and oxidative spoilage reactions occur intensively. Active packaging can overcome these limitations by providing continuous release of functional additives from packaging materials to food surface, thus, maintaining only their critical concentration at the surface necessary for inhibiting the microbial growth or oxidative change (Appendini and Hotchkiss, 2002).

Controlled release systems have been developed and used extensively for pharmaceutical applications (Langer and Peppas, 1981; Leong and Langer, 1988; Brayden, 2003). Different strategies for achieving controlled release of drugs, and preparation and modeling of drug delivery systems have been reported (Langer and Peppas, 1981; Mallapragada and Peppas, 1997; Richard, 1998; Siepmann et al., 1999; Siepmann and Peppas, 2001; Arifin et al., 2006). However, the studies in the field of food packaging are very limited although controlled release of active agents through food packaging materials is an essential feature to provide

\footnotetext{
* Corresponding author. Tel.: +90 232 7506658; fax: +90 2327506645 .

E-mail address: sacidealsoy@iyte.edu.tr (S.A. Altınkaya).
}

desired concentrations on food surfaces. Han and Floros (1998) are one of the first researchers who used the concept of controlled release in food packaging applications. They suggested controlling the release kinetics of antimicrobials by using a multilayer structure which included an outer barrier layer, a matrix layer containing the active agent and a control layer. Buonocore et al. (2005) also developed multilayer films which consist of two external control layers and an inner layer containing the active agent. The same research group has also encapsulated the active compounds in a polymer matrix and tried to control the release rates by changing the concentration of cross linking agent in the matrix (Buonocore et al., 2003, 2004). Ozdemir and Floros (2003) formed a controlled release system by dispersing hydrophobic wax particles within a hydrophilic film matrix, thus, increasing hydrophobicity and tortuosity of the films against the diffusion of an antimicrobial agent. LaCoste et al. (2005) have proposed using smart blending technique to develop controlled release packaging materials.

Recently, our research group showed the suitability of using asymmetric cellulose acetate (CA) films for controlled release of lysozyme, a natural antimicrobial enzyme used in food preservation (Gemili et al., 2009). The release rate of the active agent through these types of films is controlled with the porosity and degree of asymmetry of the films which can be easily modulated by changing the film casting conditions (Altinkaya and Yenal, 2006; Gemili et al., 2009). The application of either porous or dense sides of the films on food surface is also a great advantage to control release rates in different food systems. In this study, we aimed to 


\section{Nomenclature}

Af $\quad$ area of the film $\left(\mathrm{cm}^{2}\right)$

$D$ effective diffusion coefficient of active agent in the film $\left(\mathrm{cm}^{2} / \mathrm{s}\right)$

$K \quad$ partition coefficient $\left(\mathrm{cm}^{3}\right.$ solution $/ \mathrm{cm}^{3}$ film)

$L \quad$ thickness of the film $(\mathrm{cm})$

$M_{t} \quad$ total amount of active agent desorbed from the film at anytime $t(\mathrm{~g})$
$M_{\infty} \quad$ total amount of active agent desorbed from the film at equilibrium $(\mathrm{g})$

$t$ time (sec)

$V_{\text {sol }} \quad$ volume of the solution $\left(\mathrm{cm}^{3}\right)$ show the potential of asymmetric CA films for controlled release of different functional agents, thus, we have developed films including natural antioxidants, L-ascorbic acid and L-tyrosine. These substances were selected particularly since they are powerful antioxidants with close and low molecular weights (L-ascorbic acid: $176.1 \mathrm{~g} / \mathrm{mol}$, L-tyrosine: $181.2 \mathrm{~g} / \mathrm{mol}$ ), but very different hydrophilic/hydrophobic properties which allow using them in various food systems. The ascorbic acid is a highly polar, acidic compound whose antioxidant activity comes from its reducing power related to its 2,3-enediol moiety (Gregory, 1996). It is used frequently during curing of meats and minimal processing of fresh fruits and vegetables to inhibit enzymatic browning catalyzed by polyphenoloxidase (PPO) enzyme (Gregory, 1996; Vamos-Vigyazo, 1981). On the other hand, L-tyrosine is an antioxidant amino acid which has amphiphilic properties due to its polar and apolar groups. Similar to the other aromatic amino acids, phenylalanine and tryptophan, the antioxidant activity of L-tyrosine is due to its side chain which is capable to donate protons to free radicals (Rajapakse et al., 2005). The L-tyrosine is not commercially used in food applications. However, there is a great interest in food industry to use antioxidant proteins, peptides and amino acids as food antioxidants (Arcan and Yemenicioglu, 2007).

\section{Materials}

L-ascorbic acid and L-tyrosin were purchased from Merck (Darmstadt, Germany). ABTS (2,2-azino-bis-(3-ethylbenz-thiazoline-6-sulfonic acid)) was purchased from Sigma Chem. Co. (St. Louis, MO, USA). Trolox (( \pm )-6-hydroxy-2,5,7,8-tetra-methylchromane-2-carboxylic acid) was purchased from Fluka (Switzerland). Cellulose acetate with a molecular weight of 50,000 and acetyl content of $39.8 \%$ was obtained from Eastman (Kingsport, TN, USA). Acetone (99\%) was obtained from Merck (Darmstadt, Germany).

\section{Methods}

\subsection{Preparation of the films}

To prepare the films, cellulose acetate (CA) dissolved in acetone and antioxidant agents dissolved in distilled water were mixed in a tightly closed and sealed bottle for $30 \mathrm{~min}$ at $920 \mathrm{rpm}$. The weight percentages of acetone and antioxidants in the solution were kept constant at $80 \%$ and $1.5 \%$, respectively, while CA and water concentrations were changed to obtain films with different morphologies. The mixed solution was then cast on a polypropylene substrate by using an automatic film applicator (Sheen, Automatic film applicator-1133N, Kigston, England) at a speed of $100 \mathrm{~mm} / \mathrm{s}$. The wet thicknesses of the films were adjusted by a four-sided film applicator with a gap size of 300 microns. The cast film was then immediately transferred into an environmental chamber (Siemens, Simatic OP7, Massa Martana, Italy) and dried for $30 \mathrm{~min}$ at $25^{\circ} \mathrm{C}$ and $40 \%$ relative humidity.

\subsection{Release tests}

The release tests were conducted by using a hand made glass apparatus for both dense and porous sides of the films. In this apparatus, film samples ( $5 \mathrm{~cm}$ in diameter) were squeezed between two glass plates. One of the plates has a circular hole with a diameter of $4 \mathrm{~cm}$ at the center, and in order to contact just one surface of the film with water, two silicon o-rings (full diameter: $6 \mathrm{~cm}$, inside diameter: $4 \mathrm{~cm}$ ) were placed on both sides of the films. The details of the apparatus were given by Gemili et al. (2009). For release studies, the apparatus was placed into a glass Petri dish (10 cm in diameter) containing $50 \mathrm{~mL}$ distilled water at $4{ }^{\circ} \mathrm{C}$ and stirred magnetically at $240 \mathrm{rpm}$ with a $2 \mathrm{~cm}$ long Teflon coated rod. To prevent evaporation during release period, the petri dishes were covered tightly first with parafilms and then with their glass lids. The antioxidant activities in release medium were monitored by taking $0.4 \mathrm{~mL}$ samples at different time periods and measuring activity three times by using ABTS decolorization assay given in Section 3.3. The release tests were continued until equilibrium condition is reached.

\subsection{Determination of soluble antioxidant activity}

Based on free radical-scavenging activity, the antioxidant activity was determined as described in Re et al. (1999), by using ABTS free radical solution prepared by oxidation with potassium persulfate. The reaction mixtures for the measurements were formed by mixing $0.1 \mathrm{ml}$ of sample from release test medium and $2.4 \mathrm{ml}$ of ABTS free radical solution diluted with phosphate buffer solution (initial absorbance was almost 0.7 at $734 \mathrm{~nm}$ ). The discoloration of dark blue-colored ABTS free radical by the antioxidant was monitored at $734 \mathrm{~nm}$ for $6 \mathrm{~min}$ at $30^{\circ} \mathrm{C}$. Percentage inhibitions of ABTS free radical were then calculated and converted to trolox (a standard antioxidant which is vitamin E analogue) equivalents by using standard curves. The values obtained were divided by the area of the films to determine released antioxidant activity as $\mu$ mole trolox per $\mathrm{cm}^{2}$. All measurements were performed in triplicate.

\subsection{Determination of immobilized antioxidant activity}

Following the release tests conducted in distilled water, the free radical-scavenging activity of antioxidant agents trapped in the films was also determined. For this purpose, the films were cut into two pieces and each piece was then placed into different Petri dishes containing $25 \mathrm{ml}$ of ABTS free radical solution. The reactions were conducted in an incubator at $30^{\circ} \mathrm{C}$ under continuous magnetic stirring and the decrease in absorbance at $734 \mathrm{~nm}$ was monitored periodically. Percentage inhibition of ABTS free radical after 60 min was then calculated and converted to trolox equivalents. The immobilized antioxidant activity was reported as $\mu$ mole trolox per $\mathrm{cm}^{2}$. All measurements were performed in triplicate. 


\subsection{Morphological characterization of the films}

A scanning electron microscope (SEM) (Philips XL-30S FEG, FEI Company, Eindhoven, The Netherlands) was used to characterize the morphology of the films. Film samples were coated with gold palladium using a Magnetron Sputter Coating Instrument (VG Microtech, England). Two pictures were taken for each film. After the SEM pictures were taken, total thickness, dense skin layer thickness, porosity, and pore size of the films were measured with the aid of a software (Scandium universal SEM image platform) in the computer connected to the SEM. Five to 10 measurements were taken for each film to determine total and dense skin layer thicknesses. Two porosity measurements were conducted for each type of film. For the pore size measurement, almost 30 pores were selected in each SEM picture and the diameters of the pores were measured. The porosities (\%) were measured automatically by differentiating the colors of porous and non-porous areas of the films. The thickness of the dense skin layers was measured automatically by selecting these clearly distinguishable regions manually.

\subsection{Mechanical properties of the films}

The tensile strength of the films was measured with Testometric M500-100kN (Lancashire, England) testing machine in accordance with ASTM D 882-02 standard. Samples $5 \mathrm{~mm}$ in width and $50 \mathrm{~mm}$ in gauge length were strained at a constant rate of $0.25 \mathrm{~mm} / \mathrm{min}$ until failure. At least, five specimens were used for the tests and a plot of stress versus strain was utilized to calculate the tensile properties of the films.

\subsection{Determination of diffusion coefficients of $L$-tyrosine and $L$-ascorbic acid}

To determine diffusion coefficient of antioxidants, Fick's second law was used which describes the change in the concentration of antioxidant agents in the films with respect to time and position. To be able to use Fick's second law, it was assumed that there is no chemical reaction between the active compound and the film, mass transfer in the film takes place only by diffusion and diffusion coefficient of active compound in the film, $D$, is constant. A solution of Fick's second law along with one initial and two boundary conditions is presented in a classical book of Crank (1975). The most practical expression obtained from this solution which gives the total amount of active compound desorbed from the film at any time $t, M_{t}$, normalized with respect to the amount desorbed at equilibrium, $M_{\infty}$, is given below:

$\frac{M_{t}}{M_{\infty}}=1-\sum_{n=1}^{\infty} \frac{2 \alpha(1+\alpha)}{1+\alpha+\alpha^{2} q_{n}^{2}} \exp \left(-D q_{n}^{2} t / L^{2}\right)$

where $\alpha=\frac{V_{s o l}}{K A_{f} L}$, and the $q_{n}$ s are the non-zero positive roots of $\tan q_{n}=-\alpha q_{n}$. To determine the diffusion coefficients of antioxidants, the antioxidant activity data obtained during release tests were first converted to concentration data by using calibration curves formed for L-tyrosine and L-ascorbic acid. Then, the diffusivities were calculated iteratively by minimizing the difference between Eq. (1) given above and experimental uptake curves.

\subsection{Statistical analysis}

The statistical analysis was conducted by evaluating data with analysis of variance (ANOVA). Values are significantly different at $p<0.05$ as determined by Fisher's protected least significant difference.

\section{Results and discussions}

\subsection{The morphology of the films}

Recently, our research group successfully developed asymmetric CA films for controlled release of antimicrobial enzyme lysozyme by dry phase inversion technique (Gemili et al., 2009). This technique allows to create film morphologies ranging from dense to highly asymmetric and porous ones by changing film preparation conditions. In this work, we have used the same technique to prepare CA films containing low molecular weight natural antioxidants, L-ascorbic acid and L-tyrosine, and controlled their release rates by varying the porosity and degree of asymmetry of the films. To control such morphological features, we have changed the composition of the film forming solution. Fig. 1a-c illustrate the influence of the initial casting composition on the structure of L-ascorbic acid containing films through SEM pictures. In these pictures, the dense and porous surfaces exist on top and bottom sides of the double arrows which show the total thickness of the films. Characteristics of these films determined from the analysis of SEM images were also listed in Table 1 . As expected, both Lascorbic acid and L-tyrosine containing films prepared with the lowest CA concentration (5\%) have the highest porosities including macrovoids, with an immeasurable thin dense skin layer at the top surfaces. On the other hand, increasing $\mathrm{CA}$ /acetone ratio in the casting solution leads to the formation of less porous structure with thicker dense skin layers and reduced pore sizes. The results in Table 1 indicate that the antioxidant type caused significant differences in the porosity of the films while the pore size and dense skin layer thicknesses were found to be statistically different only for the films prepared with $10 \%$ and $15 \%$ CA, respectively.

\subsection{Effect of film composition on released antioxidant activity}

To determine the effect of film composition on released antioxidant activity, porous or dense surfaces of CA films were brought into contact with distilled water. The release tests were conducted at $+4{ }^{\circ} \mathrm{C}$ since the objective of this work is to develop active packaging materials for refrigerated foods. Figs. 2 and 3 show the increase of antioxidant activity in distilled water because of L-ascorbic acid and L-tyrosine release from the films, respectively. Although both antioxidants have similar molecular weights, the L-tyrosine releases into water much more slowly than the L-ascorbic acid. The L-tyrosine is considerably more hydrophobic than the highly polar L-ascorbic acid. Therefore, its affinity to hydrophobic CA films and a delay in the release rate of this compound is quite expected. Moreover, it is also likely that the six carbon aromatic ring of L-tyrosine which is bulkier than that of four carbon ring of ascorbic acid faces greater diffusion barrier effect due to higher tortuosity of the film. In most films, antioxidant release from porous surfaces occurred more rapidly than that from dense surfaces. The L-tyrosine containing films prepared with 5\% CA gave almost 2.5 -fold higher maximum released antioxidant activity than similar films containing L-ascorbic acid (Table 2). This occurred due to almost 4-fold greater antioxidant capacity of L-tyrosine than L-ascorbic acid. According to the slopes of inhibition vs. concentration curves obtained for the antioxidants, $1 \mu$ mole of L-ascorbic acid and L-tyrosine equal to 0.29 and $1.18 \mu$ mole of trolox, respectively (Fig. 4). When the porosity of the films reduced by increasing their CA content, the maximum antioxidant activity of L-ascorbic acid released into solution was found to be higher. This suggests high diffusion rate of L-ascorbic acid through much more dense films with reduced pore size and porosity, but trapping of significant amount of L-tyrosine in these dense films. The maximum L-tyrosine antioxidant activities released from dense and porous 

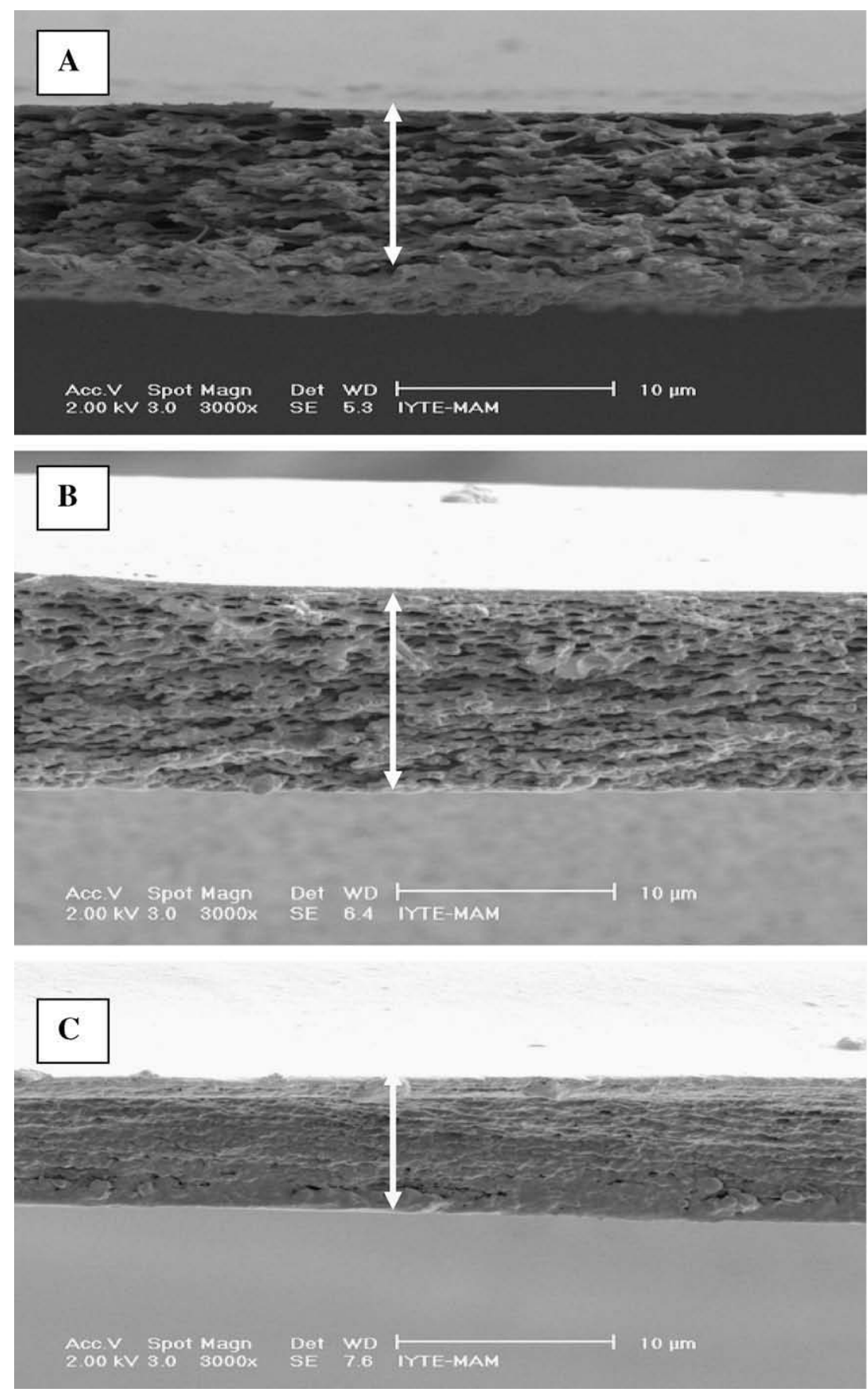

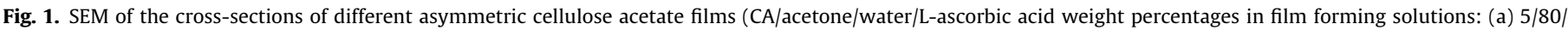
13.5/1.5, (b) 10/80/8.5/1.5, and (c) 15/80/3.5/1.5, Magnifications, $3000 \times$, upper sides of the films are the dense surfaces, while, bottom sides are porous surfaces).

surfaces were significantly different except from the film prepared with $5 \%$ CA. This can be explained by physical hindrance of small pores present on the dense surfaces against the large aromatic ring structure of L-tyrosine, consequently, its trapping within the matrix. In the case of L-ascorbic acid, the use of dense and porous surfaces of only $15 \%$ CA containing films caused significant difference in maximum antioxidant release into the solution.

\subsection{Effect of film composition on immobilized antioxidant activity}

Following release tests in distilled water, all films were also tested for immobilized antioxidant activity to estimate the amount of antioxidant trapped or bind within their matrix. In general,
L-tyrosine containing films showed greater immobilized antioxidant activity than L-ascorbic acid containing films (Table 3 ). The films prepared with 5\% CA and containing L-tyrosine showed similar immobilized antioxidant activities when their porous or dense surfaces were employed in the release tests before activity measurements. In contrast, the immobilized antioxidant activities of the films containing $10 \%$ and $15 \%$ CA were found to be significantly higher $(p<0.05)$ when the films were exposed to release test with their dense surfaces. Apparently, this occurs due to more trapped or bind L-tyrosine remained in this case. In contrast, in the case of L-ascorbic acid, the immobilized activities were measured significantly higher when the porous rather than the dense surfaces of the films were used in the release tests $(p<0.05)$. The L-ascorbic 
Table 1

Morphological characteristics of antioxidant containing cellulose acetate films.

\begin{tabular}{|c|c|c|c|c|}
\hline Composition (w\%) ${ }^{\mathrm{a}}$ & Total thickness $(\mu \mathrm{m})^{\mathrm{b}}$ & Dense layer thickness $(\mu \mathrm{m})^{\mathrm{c}}$ & Pore size $(\mu \mathrm{m})^{\mathrm{c}}$ & Porosity $(\%)^{\mathrm{c}}$ \\
\hline \multicolumn{5}{|c|}{ L-ascorbic acid containing films } \\
\hline $5 / 80 / 13.5 / 1.5$ & $11.26 \pm 0.8$ & $0 \mathrm{~b}$ & $1.28 \pm 0.09 \mathrm{~b}$ & $47.19 \pm 0.23 a$ \\
\hline $10 / 80 / 8.5 / 1.5$ & $12.54 \pm 0.58$ & $0.82 \pm 0.06 \mathrm{a}$ & $0.53 \pm 0.03 a$ & $35.28 \pm 2.5 b$ \\
\hline $15 / 80 / 3.5 / 1.5$ & $9.74 \pm 0.69$ & $1.73 \pm 0.12 c$ & $0.40 \pm 0.03 \mathrm{a}$ & $8.48 \pm 0.6 c$ \\
\hline \multicolumn{5}{|c|}{ L-tyrosine containing films } \\
\hline $5 / 80 / 13.5 / 1.5$ & $13.94 \pm 0.93$ & $0 \mathrm{~b}$ & $1.15 \pm 0.17 b$ & $54.43 \pm 1.68 d$ \\
\hline $10 / 80 / 8.5 / 1.5$ & $10.87 \pm 3.47$ & $0.83 \pm 0.23 a$ & $0.80 \pm 0.05 c$ & $25.83 \pm 0.49 \mathrm{e}$ \\
\hline $15 / 80 / 3.5 / 1.5$ & $10.20 \pm 0.65$ & $3.52 \pm 0.52 \mathrm{~d}$ & $0.50 \pm 0.04 a$ & $16.28 \pm 5.94 \mathrm{f}$ \\
\hline
\end{tabular}

a Weight percentages of $\mathrm{CA} /$ acetone/water/antioxidant agent in film forming solutions.

b No statistically significant differences exist for values in this column $(p>0.05)$.

${ }^{c}$ Different letters in each column indicate statistically significant differences $(p<0.05)$.

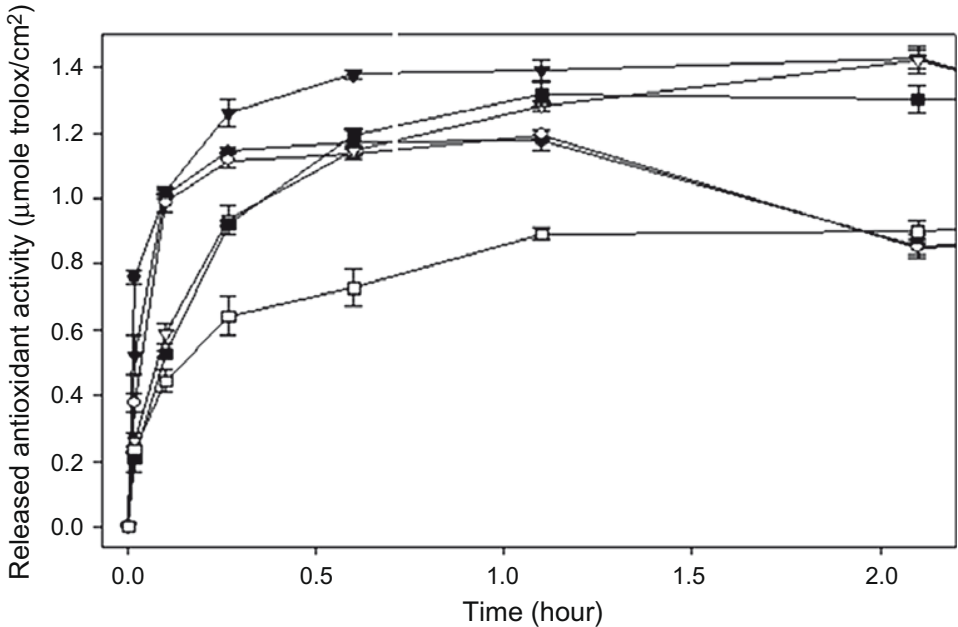

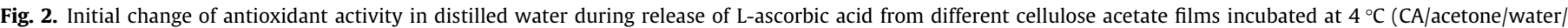

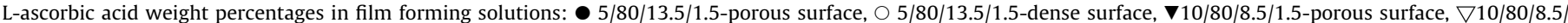
1.5-dense surface, $\boldsymbol{\square}$ 15/80/3.5/1.5-porous surface, and $\square$ 15/80/3.5/1.5-dense surface).

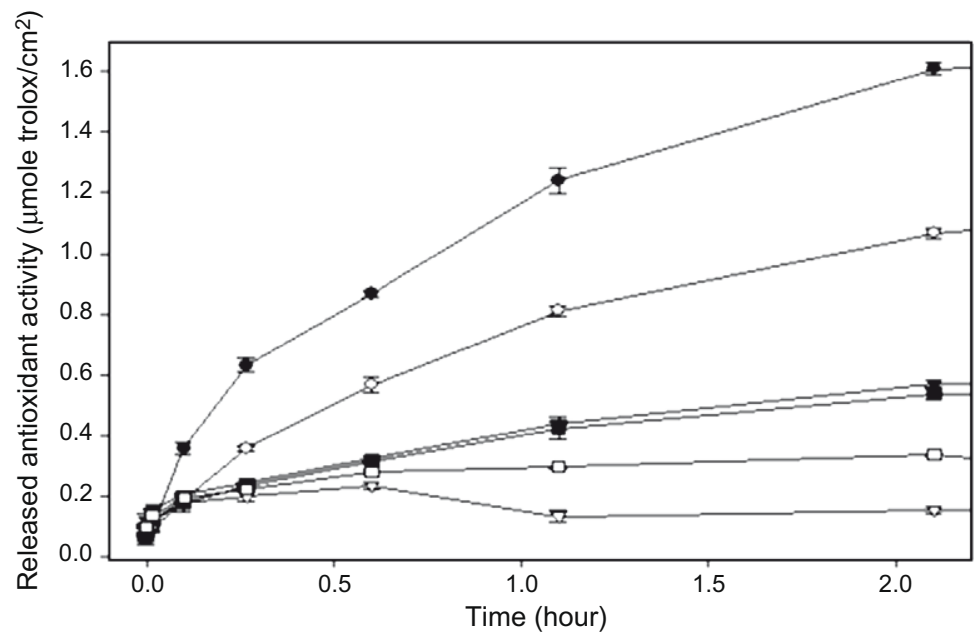

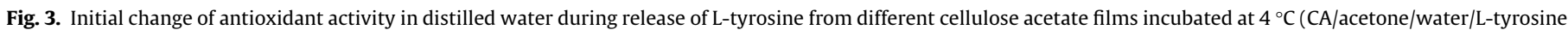

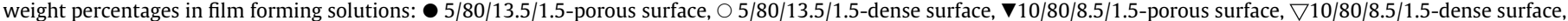
口 15/80/3.5/1.5-porous surface, and $\square$ 15/80/3.5/1.5-dense surface).

acid released readily from both dense and porous surfaces of the films. Therefore, no significant gradient in concentration of trapped antioxidant occurs through dense to porous sides of the films. In this case, it is expected to have higher immobilized L-ascorbic acid antioxidant activity on porous sides due to their greater contact area with the free radical solution. 
Table 2

Antioxidant activities obtained form different CA films during release tests.

\begin{tabular}{|c|c|c|c|}
\hline Composition (w\%) ${ }^{\mathrm{a}}$ & Film surface & Maximum released antioxidant activity-reaction period $6 \min \left(\mu \text { mole trolox } / \mathrm{cm}^{2}\right)^{b}$ & Recovery (\%) \\
\hline \multicolumn{4}{|c|}{ L-ascorbic acid containing films } \\
\hline \multirow[t]{2}{*}{$5 / 80 / 13.5 / 1.5$} & Porous & $1.18 \pm 0.03(36)^{\mathrm{c}} \mathrm{b}$ & 90 \\
\hline & Dense & $1.19 \pm 0.01(66) b$ & 85 \\
\hline \multirow[t]{2}{*}{$10 / 80 / 13.5 / 1.5$} & Porous & $1.43 \pm 0.03(126) \mathrm{a}$ & 95 \\
\hline & Dense & $1.42 \pm 0.04(126) \mathrm{a}$ & 87 \\
\hline \multirow[t]{2}{*}{$15 / 80 / 13.5 / 1.5$} & Porous & $1.33 \pm 0.09(66) \mathrm{f}$ & 82 \\
\hline & Dense & $1.01 \pm 0.03(246) c$ & 63 \\
\hline \multicolumn{4}{|c|}{ L-tyrosine containing films } \\
\hline \multirow[t]{2}{*}{$5 / 80 / 13.5 / 1.5$} & Porous & $3.05 \pm 0.04(1701) \mathrm{e}$ & 89 \\
\hline & Dense & $3.04 \pm 0.03(1520) \mathrm{e}$ & 68 \\
\hline \multirow[t]{2}{*}{$10 / 80 / 13.5 / 1.5$} & Porous & $1.16 \pm 0.01(1698) b$ & 26 \\
\hline & Dense & $0.34 \pm 0.01(1455) \mathrm{d}$ & 8.5 \\
\hline \multirow[t]{2}{*}{$15 / 80 / 13.5 / 1.5$} & Porous & $1.02 \pm 0.04(1464) c$ & 24 \\
\hline & Dense & $0.33 \pm 0.00(126) d$ & 15 \\
\hline
\end{tabular}

a Weight percentages of $\mathrm{CA} /$ acetone/water/antioxidant agent in film forming solutions.

b Different letters in column indicate statistically significant differences $(p<0.05)$.

c Incubation time (minute) to reach maximum antioxidant activity during release tests.

d (Maximum released antioxidant activity/Antioxidant activity incorporated) $\times 100$.

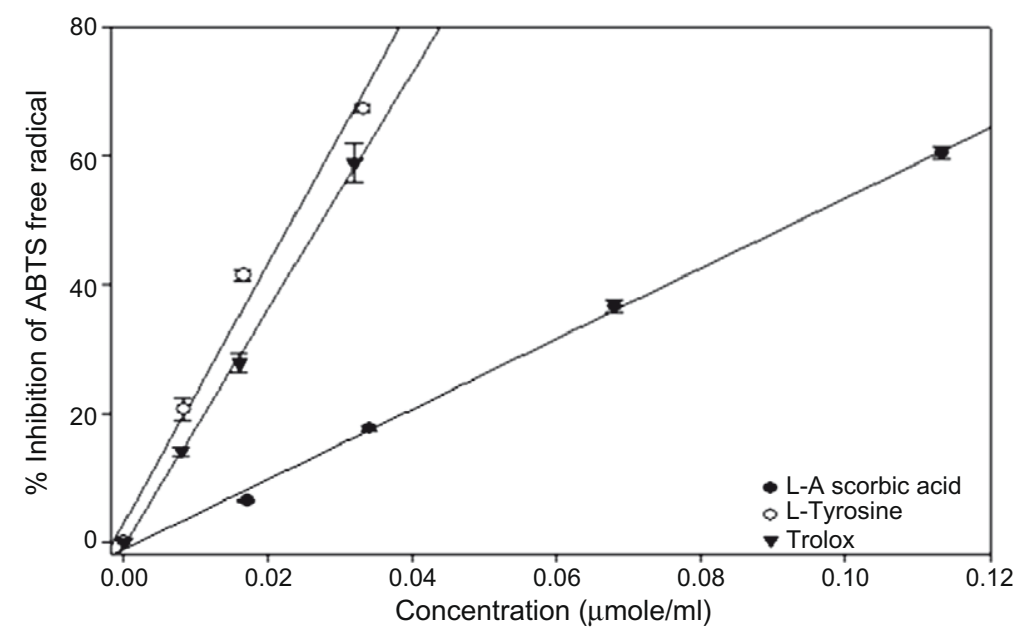

Fig. 4. Antioxidant activities of L-ascorbic acid and L-tyrosine compared to standard antioxidant trolox.

Table 3

Immobilized antioxidant activities of films after release tests.

\begin{tabular}{|c|c|c|c|}
\hline \multirow[t]{2}{*}{ Composition (w\%) } & \multirow[t]{2}{*}{ Film surface used in release test } & \multicolumn{2}{|c|}{ Immobilized antioxidant activity-reaction period $60 \mathrm{~min}\left(\mu \text { mole trolox } / \mathrm{cm}^{2}\right)^{\mathrm{b}}$} \\
\hline & & L-ascorbic acid containing film & L-tyrosine containing film \\
\hline \multirow[t]{2}{*}{$5 / 80 / 13.5 / 1.5$} & Porous & $1.10 \pm 0.04 \mathrm{a}$ & $2.04 \pm 0.04 b c$ \\
\hline & Dense & $0.75 \pm 0.00 \mathrm{~d}$ & $2.09 \pm 0.02 \mathrm{bc}$ \\
\hline \multirow[t]{2}{*}{$10 / 80 / 13.5 / 1.5$} & Porous & $1.18 \pm 0.36 \mathrm{a}$ & $1.82 \pm 0.02 c$ \\
\hline & Dense & $0.45 \pm 0.13 \mathrm{e}$ & $2.14 \pm 0.07 b$ \\
\hline \multirow[t]{2}{*}{$15 / 80 / 13.5 / 1.5$} & Porous & $1.91 \pm 0.09 \mathrm{bc}$ & $1.83 \pm 0.02 c$ \\
\hline & Dense & $1.06 \pm 0.15 a$ & $2.11 \pm 0.02 b$ \\
\hline
\end{tabular}

${ }^{\text {a }}$ Weight percentages of $\mathrm{CA} /$ acetone/water/antioxidant agent in film forming solutions.

b Different letters in columns (evaluated together) indicate statistically significant differences $(p<0.05)$.

\subsection{Effect of film composition on diffusion coefficients of antioxidants}

The normalized releases of L-tyrosine and L-ascorbic acid from CA films into water at $4{ }^{\circ} \mathrm{C}$ were given in Figs. 5 and 6 , respectively. These curves were obtained by plotting the ratio of the amount of the antioxidant agents released at time $t, M_{t}$, to the total amount released when equilibrium is reached, $M_{\infty}$. Table 4 also listed the effective diffusion coefficients of the antioxidant agents and their partition coefficients calculated from the normalized release curves using Eq. (1) and the equilibrium condition, respectively. In general, the diffusion coefficient of L-tyrosine is smaller than that of L-ascorbic acid. In most cases, the diffusivities were higher when the porous side of the films was in contact with water. The diffusion rates of L-ascorbic acid through either surface of the film prepared with 5\% CA were found same since this film has a highly porous and almost a symmetric structure. Similar diffusivities were also obtained for L-tyrosine at each surface of the film cast with $15 \%$ CA. This film has a highly dense structure where the 

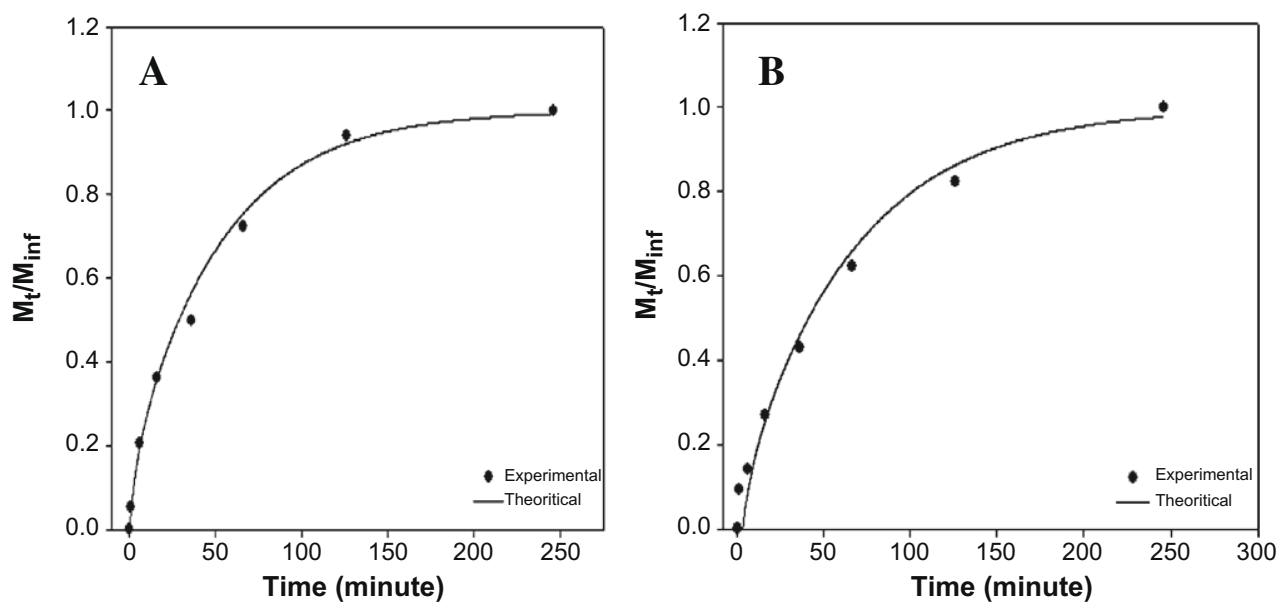

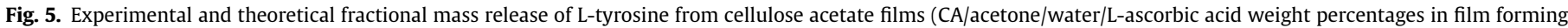
solutions: (A) 5/80/13.5/1.5-porous surface and (B) 5/80/13.5/1.5-dense surface).
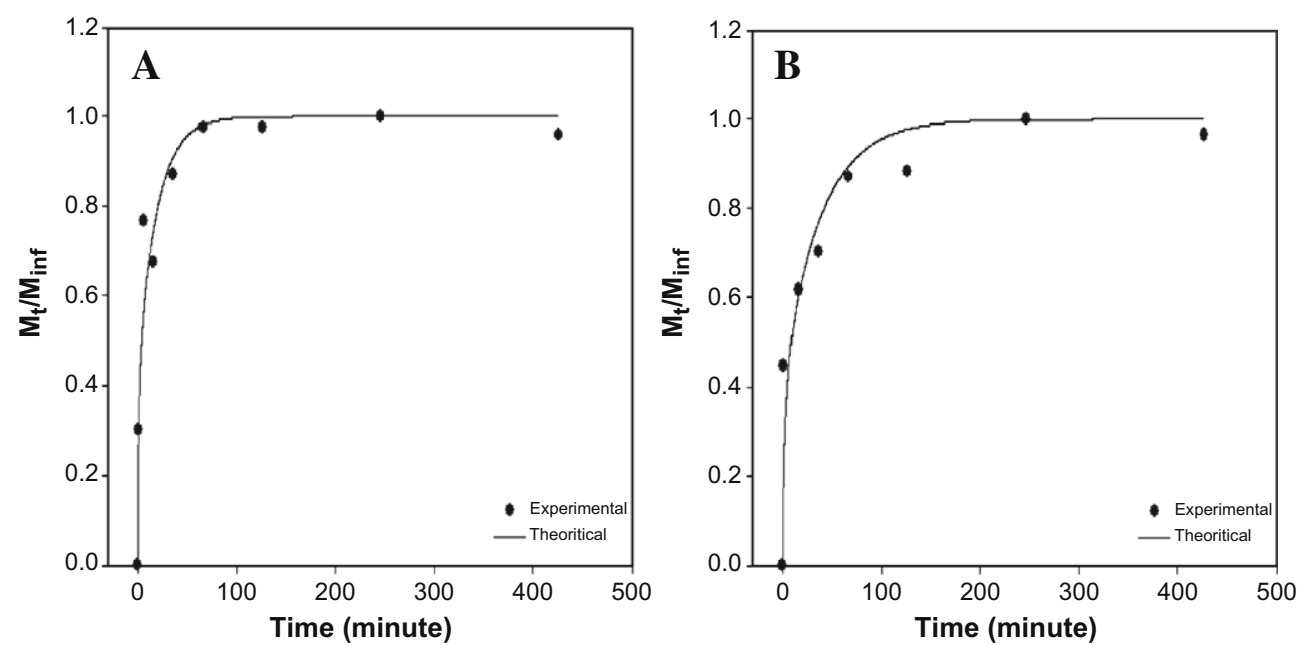

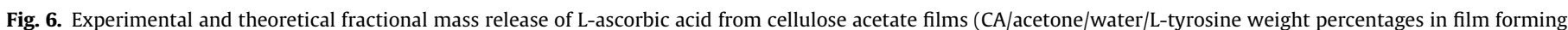
solutions: (A) 15/80/3.5/1.5-porous surface and (B) 15/80/3.5/1.5-dense surface).

Table 4

Effective diffusion and partition coefficient of antioxidant agents in different cellulose acetate films.

\begin{tabular}{llcl}
\hline Composition (w\%) & Film surface & Partition coefficient & $D\left(\mathrm{~cm}^{2} / \mathrm{s}\right)$ \\
\hline \multicolumn{2}{l}{ L-ascorbic acid containing films } & & \\
$5 / 80 / 13.5 / 1.5$ & Porous & 378 & $15.0 \times 10^{-10}$ \\
& Dense & 611 & $15.0 \times 10^{-10}$ \\
$10 / 80 / 8.5 / 1.5$ & Porous & 169 & $15.0 \times 10^{-10}$ \\
& Dense & 483 & $8.33 \times 10^{-10}$ \\
$15 / 80 / 3.5 / 1.5$ & Porous & 893 & $3.33 \times 10^{-10}$ \\
& Dense & 2439 & $1.67 \times 10^{-10}$ \\
\multicolumn{5}{l}{-tyrosine containing } & films & & \\
$5 / 80 / 13.5 / 1.5$ & Porous & 343 & $2.50 \times 10^{-10}$ \\
& Dense & 1371 & $2.00 \times 10^{-10}$ \\
$10 / 80 / 8.5 / 1.5$ & Porous & 10,464 & $1.00 \times 10^{-10}$ \\
& Dense & 39,327 & $0.17 \times 10^{-10}$ \\
$15 / 80 / 3.5 / 1.5$ & Porous & 12,553 & $0.83 \times 10^{-10}$ \\
& Dense & 22,344 & $0.83 \times 10^{-10}$ \\
\hline
\end{tabular}

a Weight percentages of $\mathrm{CA} /$ acetone/water/antioxidant agent in film forming solutions.

fraction of dense skin layer is almost $35 \%$ of total thickness, thus, the bulky L-tyrosine diffuses at the same rate when the film surfaces in contact with water were changed. It should also be noted that the diffusivities of antioxidants decreased with the increased CA content of the films due to a change in the structure of the films from highly porous and symmetric to dense and asymmetric ones. An unexpectedly low diffusivity of L-tyrosine was obtained through the dense side of the film prepared with $10 \%$ CA. This may be explained by effective blocking of pores on the dense surface by L-tyrosine molecules diffused from porous to dense side and trapped within these pores. Partition coefficients of L-tyrosine were found higher than those of L-ascorbic acid due to higher affinity of hydrophobic aromatic ring of this antioxidant to CA which has also hydrophobic nature.

\subsection{Effect of film composition on mechanical properties of the films}

The mechanical properties of the films were given in Table 5 . The results indicate that for each film type tensile strength and Young's modulus values increased significantly $(p<0.05)$ with increasing CA content in initial film forming solution. This is due to reduced porosities, pore sizes and the change in film structures from porous to dense ones. Except the control films, elongation values of the films did not correlate with the CA content. The results in Table 5 indicate that compared with the control films, 
Table 5

Mechanical properties of developed cellulose acetate films.

\begin{tabular}{|c|c|c|c|}
\hline Composition (w\%) & Elongation $(\%)^{\mathrm{c}}$ & Tensile strength $(\mathrm{MPa})^{\mathrm{c}}$ & Young's modulus $(\mathrm{MPa})^{\mathrm{c}}$ \\
\hline \multicolumn{4}{|l|}{ Control films ${ }^{\mathrm{b}}$} \\
\hline $5 / 80 / 15 / 0$ & $1.76 \pm 0.41 \mathrm{~d}$ & $10.37 \pm 1.61 \mathrm{c}$ & $257.52 \pm 46.84 \mathrm{a}$ \\
\hline $10 / 80 / 10 / 0$ & $4.02 \pm 1.19 \mathrm{a}$ & $37.05 \pm 1.70 \mathrm{a}$ & $1214.81 \pm 63.24 \mathrm{c}$ \\
\hline $15 / 80 / 5 / 0$ & $12.00 \pm 1.58 \mathrm{f}$ & $86.31 \pm 3.75 b$ & $2068.33 \pm 94.73 d$ \\
\hline \multicolumn{4}{|c|}{ L-ascorbic acid containing films } \\
\hline $5 / 80 / 13.5 / 1.5$ & $3.32 \pm 0.78$ ace & $6.18 \pm 1.54 \mathrm{c}$ & $165 \pm 41 \mathrm{a}$ \\
\hline $10 / 80 / 8.5 / 1.5$ & $2.55 \pm 0.66 \mathrm{de}$ & $30.43 \pm 6.09 a$ & $1535 \pm 204 b$ \\
\hline $15 / 80 / 3.5 / 1.5$ & $6.43 \pm 2.69 \mathrm{~g}$ & $86.20 \pm 15.23 b$ & $2527 \pm 261 \mathrm{e}$ \\
\hline \multicolumn{4}{|c|}{ L-tyrosine containing films } \\
\hline $5 / 80 / 13.5 / 1.5$ & $0.90 \pm 0.35 \mathrm{bd}$ & $2.50 \pm 1.04 \mathrm{c}$ & $274 \pm 48 a$ \\
\hline $10 / 80 / 8.5 / 1.5$ & $3.64 \pm 0.23$ ae & $36.39 \pm 6.84 a$ & $1408 \pm 111 \mathrm{~d}$ \\
\hline $15 / 80 / 3.5 / 1.5$ & $2.03 \pm 0.26 \mathrm{bcd}$ & $73.06 \pm 9.48 \mathrm{~d}$ & $2716 \pm 173 \mathrm{f}$ \\
\hline
\end{tabular}

a Weight percentages of $\mathrm{CA} /$ acetone/water/antioxidant agent in film forming solutions.

b Data of control films prepared with the same procedure explained in this work were obtained from our previous study (Gemili et al., 2009).

c Different letters in each column indicate statistically significant differences $(p<0.05)$.

the use of L-ascorbic acid did not cause any significant change in the tensile strength, while in the case of L-tyrosine significant reduction was observed in the tensile strengths of films cast with $15 \%$ CA. Young's modulus of the films were significantly affected by the addition of antioxidants except the most porous films containing the lowest CA content. The use of L-tyrosine caused a significant reduction in elongation only at $15 \%$ CA content, while significant changes, a reduction or increase, in this parameter occurred at all CA concentrations when L-ascorbic acid was used as antioxidant.

\section{Conclusion}

In conclusion, this study clearly showed the good potential of using asymmetric cellulose acetate films for controlled release of L-ascorbic acid and L-tyrosine. Changing the composition of the casting solution to control the degree of asymmetry and pore size of the films and application of either dense or porous sides of the films on food surfaces are key points to obtain desired release rates for the antioxidants, hence, to increase the quality and shelf life of the foods. Further studies are needed to develop novel food packaging applications by testing the effectiveness of the developed films on selected food surfaces.

\section{References}

Altinkaya, S.A., Yenal, H., 2006. In vitro drug release rates from asymmetricmembrane tablet coatings: prediction of phase-inversion dynamics. Biochemical Engineering Journal 28 (2), 131-139.

Appendini, P., Hotchkiss, J.H., 2002. Review of antimicrobial food packaging. Innovative Food Science and Emerging Technologies 3 (2), 113-126.

Arcan, I., Yemenicioğlu, A., 2007. Antioxidant activity of protein extracts from heat treated or thermally processed chickpeas and white beans. Food Chemistry 103, 301-312.

Arifin, D.Y., Lee, L.Y., Wang, C.H., 2006. Mathematical modeling and simulation of drug release from microspheres: implications to drug delivery systems. Advanced Drug Delivery Reviews 58 (12-13), 1274-1325.

Brayden, D.J., 2003. Controlled release technologies for drug delivery. Drug Discovery Today 8 (21), 976-978.
Buonocore, G.G., Conte, A., Corbo, M.R., Sinigaglia, M., Nobile, M.A., 2005. Mono- and multilayer active films containing lysozyme as antimicrobial agent. Innovative Food Science and Emerging Technologies 6 (4), 459-464.

Buonocore, G.G., Del Nobile, M.A., Panizza, A., Bove, S., Nicolais, L., 2003. Modeling the lysozyme release kinetics from antimicrobial films intended for food packaging applications. Food Engineering and Physical Properties 68 (4), 13651370.

Buonocore, G.G., Sinigaglia, M., Corbo, M.R., Bevilacqua, A., La Notte, E., Del Nobile, M.A., 2004. Controlled release of antimicrobial compounds from highly swellable polymers. Journal of Food Products 67 (6), 1190-1194.

Crank, J., 1975. The Mathematics of Diffusion, second ed. Oxford University Press, New York. pp. 41-50

Gemili, S., Yemenicioğlu, A., Altınkaya, S.A., 2009. Development of cellulose acetate based antimicrobial food packaging materials for controlled release of lysozyme. Journal of Food Engineering 90, 453-462.

Gregory, J.F., 1996. Vitamins. In: Fennema, O.R. (Ed.), Food Chemistry. Marce Dekker, Inc., New York. p. 559.

Han, J.H., Floros, J.D., 1998. Simulating diffusion model and determining diffusivity of potassium sorbate through plastic to develop antimicrobial packaging films. Journal of Food Processing and Preservation 22 (2), 107-122.

LaCoste, A., Schaich, M., Zumbrunnen, D., Yam, K.L., 2005. Advancing controlled release packaging through smart blending. Packaging Technology and Science 18 (2), 77-87.

Langer, R.S., Peppas, N.A., 1981. Present and future applications of biomaterials in controlled drug delivery systems. Biomaterials 2 (4), 201-214.

Leong, K.W., Langer, R., 1988. Polymeric controlled drug delivery. Advanced Drug Delivery Reviews 1 (3), 199-233.

Mallapragada, S.K., Peppas, N.A., 1997. Crystal dissolution-controlled release systems: I. Physical characteristics and modeling analysis. Journal of Controlled Release 45 (1), 87-94.

Ozdemir, M., Floros, J.D., 2003. Film composition effects on diffusion of potassium sorbate through whey protein films. Journal of Food Science 68, 511-516.

Rajapakse, N., Mendis, E., Jung, W., Je, J., Kim, S., 2005. Purification of a radical scavenging peptide from fermented mussel sauce and its antioxidant properties. Food Research International 38, 175-182.

Re, R., Pellegrini, N., Proteggente, A., Pannala, A., Yang, M., Rice-Evans, C., 1999. Antioxidant activity applying an improved ABTS radical cation decolorization assay. Free Radical Biology and Medicine 26, 1231-1237.

Richard, C., 1998. Mathematical modeling of controlled release from implanted drug impregnated monoliths. Pharmaceutical Science and Technology Today 1 (6), 269-276.

Siepmann, J., Peppas, N.A., 2001. Modeling of drug release from delivery systems based on hydroxypropyl methylcellulose (HPMC). Advanced Drug Delivery Reviews 48 (23), 139-157.

Siepmann, J., Lecomte, F., Bodmeier, R., 1999. Diffusion-controlled drug delivery systems: calculation of the required composition to achieve desired release profiles. Journal of Controlled Release 60 (2-3), 379-389.

Vamos-Vigyazo, L., 1981. Polyphenol oxidase and peroxidase in fruits and vegetables. Critical Reviews in Food Science and Nutrition 15, 49-127. 